\title{
Morphological and Cytological Characterization of Petaloid-type Cytoplasmic Male Sterility in Camellia oleifera
}

\author{
Huan Xiong, Ping Chen, Zhoujun Zhu, Ya Chen, Feng Zou' ${ }^{1}$, \\ and Deyi Yuan ${ }^{1}$ \\ Central South University of Forestry and Technology, Key Lab of Non-wood \\ Forest Products of State Forestry Administration, Key Laboratory of \\ Cultivation and Protection for Non-Wood Forest Trees, Ministry of \\ Education, Changsha 410004, Hunan, China
}

Additional index words. oil tea, cytoplasmic male sterility, anther development, tapetum, anther vascular bundle

\begin{abstract}
Camellia oleifera is an important woody tree species in China that produces edible oil. Although sterile male $C$. oleifera plants play an important role in hybrid breeding, the possible cytological characteristics of pollen abortion remain unknown. To characterize the pollen abortion process, a genic petaloid-type sterile male $C$. oleifera ' $X 1$ ' plant was investigated using a cytological method. The results showed that in malefertile plants, the anthers were full and butterfly shaped, the pollen viability was as high as $97.5 \%$, and the development of the tapetum and anther vascular bundles was normal. However, in male-sterile $C$. oleifera ' $X 1$ ', petaloidy in the anther was observed, and the pollen vitality was as low as $4.5 \%$. Pollen abortion in sterile $C$. oleifera ' $X 1$ ' anthers occurred from the microspore stage to the mature pollen period. Further cytological analyses revealed an abnormally enlarged tapetum and retarded tapetum degeneration, suggesting that insufficient nutrients were provided for microspore development. Moreover, the anther vascular bundles displayed hyperplasia, and the pollen sac area became increasingly smaller, causing most anthers to be sterile and to have few pollen grains. Taken together, the results indicate that petaloid-type male sterility in $C$. oleifera may be attributed to abnormal development of the tapetum and anther vascular bundles. The findings clarify the pollen abortion period and the cytological characteristics of petaloid-type cytoplasmic male sterility in $C$. oleifera, and lay a solid foundation for the male sterile line in $C$. oleifera hybrid breeding.
\end{abstract}

Camellia oleifera, which belongs to the genus Camellia, family Theaceae, is one of the most important woody edible oil seed crop species in China (Xiong et al., 2019). This species grows primarily south of the Yangtze River in China, including in the provinces of Hunan, Jiangxi, Guangxi, Hubei, Henan, Anhui, Zhejiang, Fujian, Guangdong, Guizhou, Yunnan, and Hainan (Gao et al., 2015a; Qin et al., 2018). The tea oil derived from this species is rich in unsaturated fatty acids and vitamin E, and is known as "Oriental olive oil" throughout the world (Gao et al., 2018). The current cultivated area of $C$. oleifera in China is $\approx 4.67$

Received for publication 28 Feb. 2019. Accepted for publication 11 Apr. 2019.

This work was supported by the National Key Research and Development Program Project of China (no. 2018YFD1000603), the Major Scientific and Technological Special Program in Hunan Province (no. 2018NK1030), and the National Forestry Public Welfare Foundation of China (no. 201504705).

1'Corresponding authors. E-mail: zoufeng06@126. com or csuftyuanyi@126.com. million ha, with an average tea oil yield of $150 \mathrm{~kg} / \mathrm{ha}$ (Wen et al., 2018). Currently present, there is a low-yield and low-profitper-hectare phenomenon in C. oleifera production, and various strategies have been developed for fixed heterosis in this plant to improve yield (Chen et al., 2011). Crossbreeding is crucial for conventional breeding of $C$. oleifera, and although $C$. oleifera is a crosspollinated plant with strong heterosis in the $F_{1}$ generation (Han et al., 2015), crossbreeding requires labor-intensive emasculation in the field. In general, male sterility is a much sought-after trait in economic crop plants because it prevents the need for hand emasculation during the production of hybrid seed (Li et al., 2010). Thus, the discovery of male-sterile plants in $C$. oleifera acting as female parents facilitates exploiting heterosis in this species.

Male sterility in plants is defined by the loss of function or degeneration of male organs resulting from genetic or physiological factors, and these plants cannot produce normal pollen or anthers (Yu et al., 2010). Male sterility is a common biological phenomenon present in many angiosperms and is found in 617 varieties or interspecific hybrids of 320 species from 162 genera within 43 families (Laser and Lersten, 1972). Male sterility can be classified as genic male sterility, cytoplasmic male sterility (CMS), or cytoplasmic-genic male sterility in higher plants according to inheritance or origin (Chen et al., 2017). Furthermore, CMS in crop species can be subdivided based on the phenotypic characteristics of the stamens, including the brown anther type and the petaloid stamen type (Kozik et al., 2012). In petaloid-type male sterility, the anther filament structure is replaced by petals or sepals (Yu et al., 2010). This petaloid type of male sterility is genetically stable, is less affected by the environment, and is easy to identify. Petaloid stability is found in many crop species, including Solanum melongena (Chen et al., 2013), Brassica juncea (Yu et al., 2010, 2014), Daucus carota (Kozik et al., 2012; Tan et al., 2017; Wolyn and Chahal, 1998; Wright et al., 1996), and Tagetes erecta (He et al., 2010; Kumar et al., 2017). In higher plants, the primary causes of CMS include abnormal microspore and tapetal cell development (Jiang et al., 2015; Luo et al., 2018; Sun et al., 2016; Xie et al., 2018), abnormal adenosine triphosphatase activity (Ji et al., 2014), and excessively high concentrations of $\mathrm{Ca}^{2+}$ (Tian et al., 1998). Because different CMS lines exhibit significant differences in phenotypic traits and sterility mechanisms, a systematic study of the sterility mechanisms in different CMS lines will provide an important reference for breeders to use these resources effectively.

A previous study reported that the malesterile line of $C$. oleifera was not completely infertile (Chen et al., 2011). In that study, the pollen grains from sterile lines displayed more shrinkage and were irregularly shaped at the mature pollen stage, the pollen exine was thin, and pollen viability was low. Nonetheless, the structural events of anther development involved in petaloid-type male sterility in $C$. oleifera remain unknown. Therefore, in our study, we examined the anther structure and pollen morphology for a cytological analysis of $C$. oleifera with petaloid-type male sterility and explored the cytological characteristics of pollen abortion to provide a theoretical basis for the application of male sterility in $C$. oleifera.

\section{Materials and Methods}

Plant materials. In late Oct. 2009, the specific resource $C$. oleifera 'Z720' was obtained from the $C$. oleifera germplasm resource garden at Majiahe Town, Zhuzhou City, Hunan Province, and its natural hybrid seeds were collected. In March 2010, the seeds were sown in the Camellia garden of Central South University of Forestry and Technology, Changsha City, Hunan Province. The garden soil is a red soil with a $\mathrm{pH}$ of 6.5. The Camellia garden is located at lat. $28^{\circ} 8^{\prime} 11^{\prime \prime} \mathrm{N}$ and long. $112^{\circ} 59^{\prime} 31^{\prime \prime} \mathrm{E}$ at an elevation of $80 \mathrm{~m}$. The annual average temperature is $17.2^{\circ} \mathrm{C}$ and the annual average precipitation is $1361.6 \mathrm{~mm}$. In Fall 2015, 
one $C$. oleifera mutant plant with a unique phenotypic trait was found in the half-sib family ' $Z 720$ '. This mutant plant exhibited petaloid-type male sterility during the flowering stage and was named ' $\mathrm{X} 1$ '. In late May 2016, propagation and preservation of the $C$. oleifera 'X1' plant were performed using high-crown grafting in the Camellia garden.

Characterization of flower organ morphology. In late Oct. 2017, the flower buds of the male sterility line ' $\mathrm{X} 1$ ' were harvested; half-sib family 'Z720' plants, which had normal petals, were used as controls. One part of the materials was photographed using a camera (Nikon 3100; Tokyo, Japan) and a stereomicroscope (Olympus SZX-16; Tokyo, Japan). Another set of samples was used for whole-anther clearing by fixation in $70 \%$ alcohol:formaldehyde:glacial acetic acid (FAA; 90:5:5, v/v). The samples were stained with hematoxylin for $15 \mathrm{~min}$, after which the staining solution was rinsed clean. The specimens were dehydrated using an alcohol gradient and were treated with methyl salicylate. The anthers were observed and photographed using an Olympus BX-51 microscope (Gao et al., 2018).

Pollen viability and pollen grain morphology. In late October 2017, flowers were collected at the balloon stage, and pollen viability was determined by an in vitro pollen germination method using $1 \%$ agar (Xiong et al., 2016). Unopened flowers were harvested and fixed in $2.5 \%$ glutaraldehyde and then dehydrated in a tertbutanol gradient for $15 \mathrm{~min}$ each. After drying, the samples were sprayed with gold particles and the morphology of the pollen grains was photographed using a scanning electron microscope (JSM-6380LV; Tokyo, Japan) (Gao et al., 2015b). For each sample, 30 pollen grains were used to determine pollen size with ImageJ (National Institutes of Health, Bethesda, MD). Significant differences between the means for pollen size and pollen viability were assessed using Duncan's multiple comparison test at $P \leq$ 0.05 .
Cytological characterization of anther development. Flower buds were harvested weekly from Oct. to Nov. 2017. One set of samples was fixed in FAA for paraffin sectioning to observe anther structure. After $24 \mathrm{~h}$, the specimens were transferred to $70 \%$ ethanol and stored at $4{ }^{\circ} \mathrm{C}$. The samples were then dehydrated with an alcohol gradient, cleared with xylene, and embedded in paraffin at $60^{\circ} \mathrm{C}$. The samples were sectioned into 8- to $10-\mu \mathrm{m}$-thick slices with a microtome (Leica RM2265; Wetzlar, Germany), stained with hematoxylin-eosin or safraninfast green, and sealed with Canadian neutral resin. Typical sections were observed and photographed using an Olympus BX-51 microscope (Zou et al., 2016). Another set of materials was used for observations of microspore morphology by transmission electron microscopy (TEM). The anthers were first fixed with $2.5 \%$ glutaraldehyde in 0.1 mol. $\mathrm{L}^{-1}$ phosphate buffer solution ( $\mathrm{pH}$ 7.2) at $4{ }^{\circ} \mathrm{C}$ for $4 \mathrm{~h}$. The samples were then washed three times with $0.1 \mathrm{~mol} \cdot \mathrm{L}^{-1}$ phosphate buffer
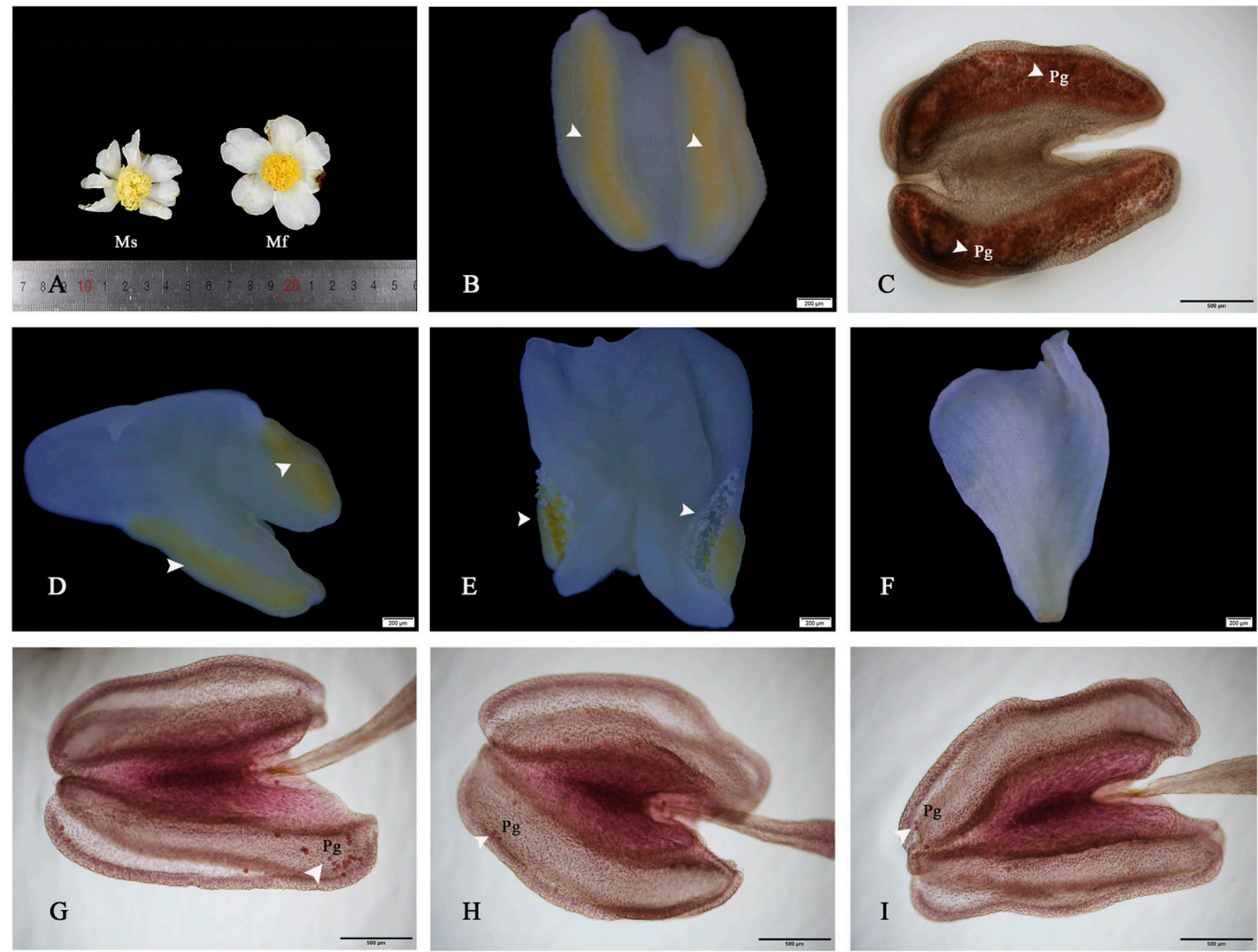

Fig. 1. Flower organ morphologies of the Camellia oleifera male sterility line 'X1' and the male-fertile half-sib family plant 'Z720'. (A) Comparison of flower morphology between 'X1' and the male-fertile half-sib family plant 'Z720'. (B) Anther morphology of the half-sib family plant 'Z720' with normal anther (C) A large number of pollen grains can be observed in the pollen sac of the half-sib family plant 'Z720'. (D, E) The anther morphology of $C$. oleifera 'X1' was partially sterile. (F) The anther morphology of $C$. oleifera 'X1' was completely sterile. (G-I) A few pollen grains can be observed in the pollen sac of $C$. oleifera 'X1'. Ms, male sterility; Mf, male fertile; Pg, pollen grain. 
solution, postfixed with $1 \%$ osmic acid in 0.1 mol. $\mathrm{L}^{-1}$ phosphate buffer solution $(\mathrm{pH} 7.2)$ at $4{ }^{\circ} \mathrm{C}$ for $12 \mathrm{~h}$, and rinsed with $0.1 \mathrm{~mol} \cdot \mathrm{L}^{-1}$ phosphate buffer and double-distilled water three times for $30 \mathrm{~min}$ each. Next, the specimens were dehydrated in an acetone gradient and embedded in Epoxy resin Epon 812. An ultramicrotome (Leica EM UC7; Wetzlar, Germany) with a diamond cutter was used to cut the tissue into 70-nm-thick sections. The sections were placed on mesh copper grids, stained with uranyl acetate and lead citrate, and air-dried. Finally, the sections were observed and photographed by TEM (Hitachi TEM 7700; Tokyo, Japan) at 80 kV (Gao et al., 2018).

\section{Results}

Characterization of flower organ morphology. The morphology of the stamens from the male sterility line 'X1' was quite different from that of the male-fertile half-sib family plant 'Z720' (Fig. 1). The stamens of the half-sib family 'Z720' were normal: The anthers were full and butterfly shaped, and their color was bright yellow. The fertile plants produced a large number of normal pollen grains (Fig. 1A-C). In contrast, the stamens of ' $\mathrm{X} 1$ ' were petaloid (Fig. 1D-F), and microscope observations revealed fewer pollen grains in the pollen sac than in malefertile anthers (Fig. 1G-I).

Pollen viability and pollen grain morphology. There was a significant difference in pollen viability between the male sterility line ('X1') and the male-fertile halfsib family plant ('Z720') (Table 1), with $97.5 \%$ obtained for the male-fertile plant (Fig. 2A) and only $4.5 \%$ for the petaloidtype male sterility ' $\mathrm{X} 1$ ' plant. There was also a large number of nongerminated pollen grains in the latter (Fig. 2C). Scanning electron microscope observation of the mature pollen grains from both plants demonstrated a nearly spherical morphology. The pollen grains were similar in size, the germinal apertures appeared to be 3-colporate (Fig. 2B and E), and the exine surface ornamentation of the pollen grains was foveolate-reticulate (Fig. 2C and F).

Cytological characterization of anther development. There were evident differences in anther development between the male sterility line ' $\mathrm{X} 1$ ' and the male-fertile halfsib family plant 'Z720' (Figs. 3 and 4), specifically with regard to the tapetum and vascular bundle of the anther. Pollen development in $C$. oleifera began at the end of meiosis with the formation of a tetrad of microspores and ended at the dehiscence of anthers when the mature pollen was released. We emphasize the three developmental stages of pollen development between the two $C$. lines in as follows: 1) tetrad, 2) microspore, and 3) pollen maturity. In both the male sterility line ' $\mathrm{X} 1$ ' and the malefertile of half-sib family plant 'Z720', a tetrad was formed normally and the cell volume of the tapetum of the anther increased significantly with the thick cytoplasm (Figs. 3A and $4 \mathrm{~A})$. The microspores were then released from the tetrads.

At the early microspore stage, the volume of microspore cells was smaller, the cell wall was thin, the cytoplasm was thick, and the nucleus was in the center of the cell of the male-fertile plants. Subsequently, the volume of the microspore increased and the cytoplasm was vacuolated to form single nuclear cells (Fig. 3B and C). As time passed, the tapetum gradually disintegrated (Fig. 3C) and the edge of the cell was blurry, without a clear outline. These cells started to degrade, and a large number of Ubisch bodies were attached to the cell surface of the tapetum (Fig. 3D). In addition, the pollen exine was fully developed (Fig. 3E and F). However, compared with the male-fertile plants, the cells of the tapetum in the male sterility line ' $\mathrm{X} 1$ ' were

Table 1. Pollen morphology and pollen viability of the Camellia oleifera male sterility line 'X1' and the male-fertile half-sib family plant 'Z720'.

\begin{tabular}{|c|c|c|c|c|}
\hline Material & Pollen morphology & Pollen grain size $(\mu \mathrm{m})^{z}$ & Surface ornamentation & Pollen viability $(\%)^{z}$ \\
\hline $\mathrm{X} 1$ & Nearly spherical & $41.99 \times 33.15 \mathrm{a}$ & Foveolate-reticulate & $4.5 \mathrm{~b}$ \\
\hline Z720 & Nearly spherical & $42.29 \times 31.76 \mathrm{a}$ & Foveolate-reticulate & $97.5 \mathrm{a}$ \\
\hline
\end{tabular}

${ }^{\mathrm{z}}$ Different letters within the same line represent significant differences at the $5 \%$ level as determined by Duncan's multiple range test; the same letter represents no significant differences.
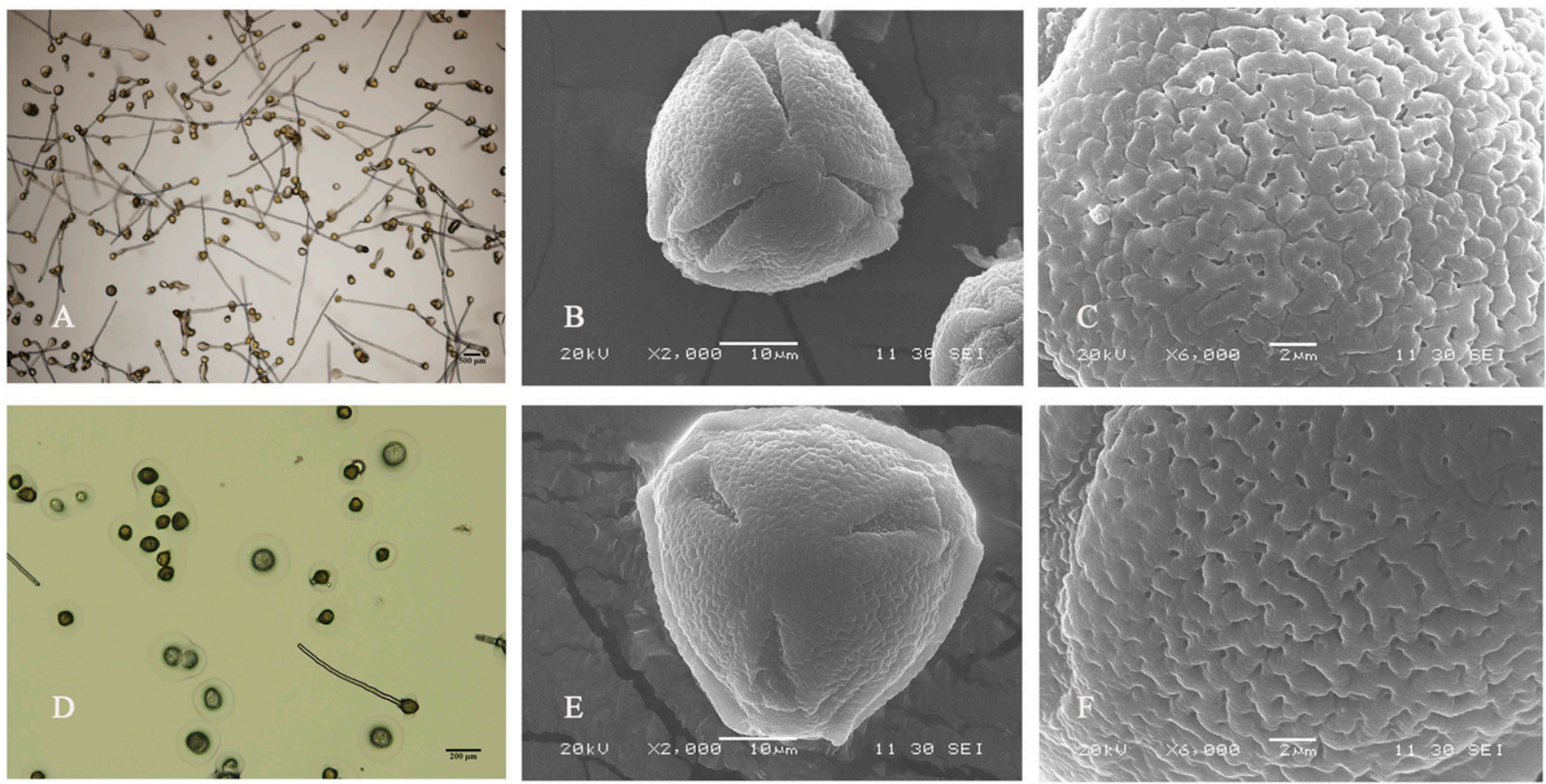

Fig. 2. Pollen viability and pollen morphology of the Camellia oleifera male sterility line 'X1' and the male-fertile half-sib family plant ' Z720'. (A) Normal pollen viability of the half-sib family plant 'Z720'. (B) Polar view of a half-sib family plant 'Z720' pollen grain. (C) Ornamentation of half-sib family plant 'Z720' pollen grains. (D) Pollen viability of the sterility line C. oleifera 'X1'. (E) Polar view of a C. oleifera 'X1' pollen grain. (F) Ornamentation of C. oleifera 'X1' pollen grains. 

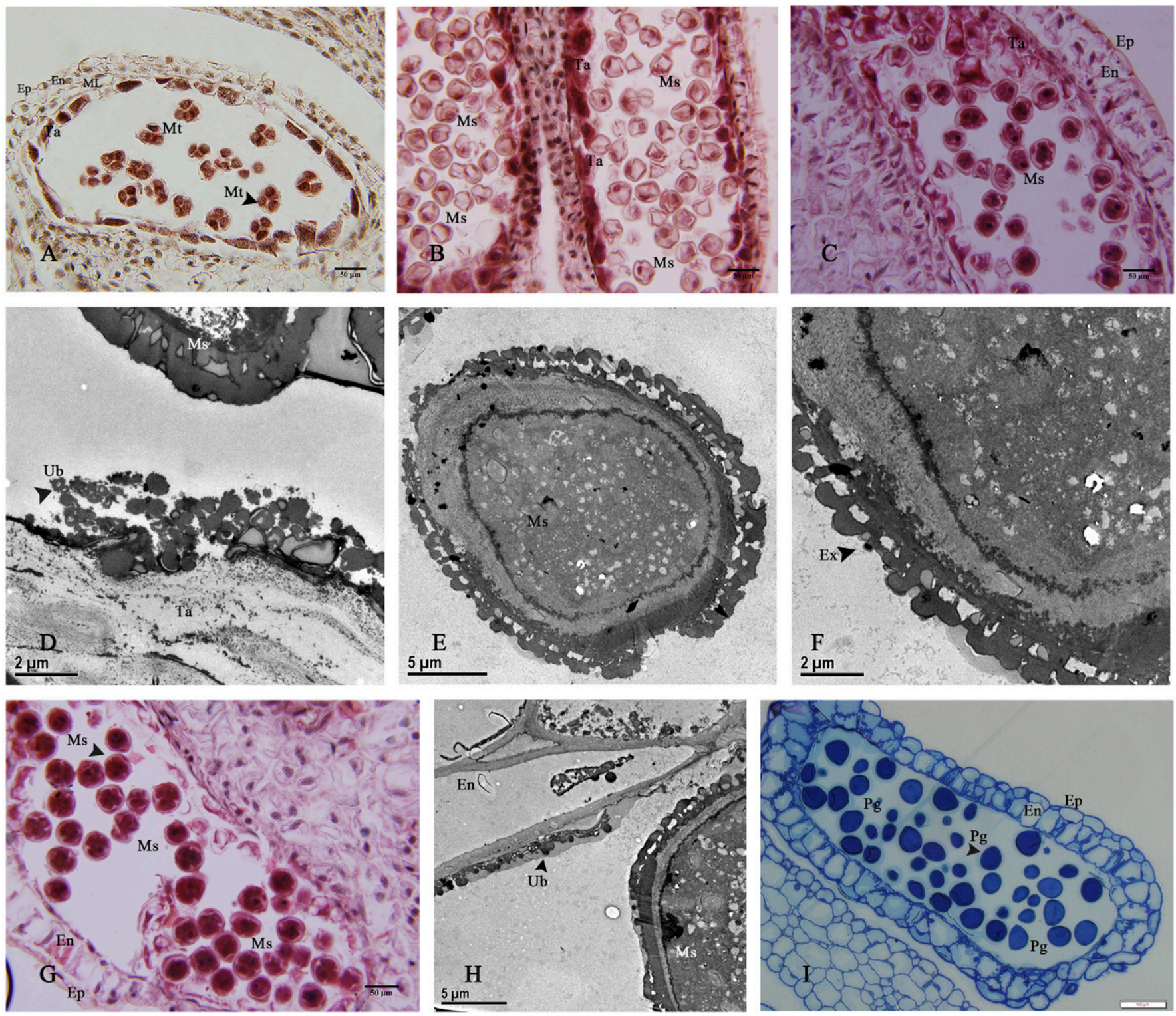

Fig. 3. Characterization of anther development in the male-fertile half-sib family plant 'Z720'. (A) Details of microspores showing tetrahedral microspore tetrads enclosed in the callose. (B) Details of anthers at the microspore cell stage. (C) Details of anthers at the uninucleate microspore stage with a stretched epidermis; fibrous, thickened endothecium; relic of middle layers; and degenerated tapetum. (D) Transmission electron microscope (TEM) observation of the disintegrating tapetum, with many Ubisch bodies attached to the surface of the cell membrane. (E) The microspore cell wall and the fully developed covering surface were fully developed under TEM observation. (F) Greater magnification of $\mathbf{E}$ showing the microspore pollen exine. (G) Details of an anther at the binucleate microspore stage, during which the tapetum gradually disappeared. (H) TEM observation of the tapetum that disappeared, and a few Ubisch bodies that were attached to the tapetum membrane. (I) Details of anthesis showing the anther wall, which comprised an epidermis, endothecium, and mature pollen grains. The tapetum had completely disintegrated and disappeared. En, endothecium; Ep, epidermis; Ex, exine; ML, middle layers; Mt, microspore tetrads; Ms, microspore; Pg, pollen grain; Ta, tapetum; Ub, Ubisch body.

hypertrophied (Fig. 4B) and the microspores were compressed (Fig. 4C). In addition, although the pollen exine of the male sterility line 'X1' developed normally (Fig. 4D and E), the tapetum did not begin to disintegrate (Fig. 4F) at this stage, and many Ubisch bodies were attached to the cell surface (Fig. 4G). When the pollen reached maturity, a larger number of pollen grains with no inclusions were observed in the pollen sac (Fig. 4H). Hence, the failure in pollen development was likely because the tapetal cells enlarged abnormally and tapetum degradation was retarded at the microspore stage; thus, not enough nutrients were provided for further pollen development.
In the male-fertile plant, the tapetum disintegrated and disappeared at the mature pollen stage, leaving only an epidermis and an endothecium (Fig. 3G). At the same time, a few Ubisch bodies were attached to the membrane of the tapetum (Fig. 3H), eventually forming two-celled pollen grains (Fig. 3I). However, in the male sterility line 'X1', abnormal anther vascular bundle development was observed during the pollen maturity stage (Fig. 4I-P). Vascular bundle hyperplasia of sterile anthers can be divided into two types: radially inward and outward. In some sterile anthers, although a small proportion of mature pollen grains were observed in the anther chambers, the vascular bundles proliferated inward continuously. In addition, the area of the pollen sacs shrank gradually (Fig. 4I and J), leading to empty anther locules (Fig. 4K and L). Instead, the vascular bundles in other anthers exhibited an abnormal structure, such as radially outward hyperplasia (Fig. 4M-P), which also caused the anther locules to become increasingly smaller, and the number of mature pollen grains thus decreased (Fig. $4 \mathrm{~N}$ and $\mathrm{O}$ ). Finally, the shape of the sterile anther changed completely and became a long strip (Fig. 4N-P). Therefore, no mature pollen grains at the end of development were present, resulting in a deformed sterile anther (Fig. 4P). 

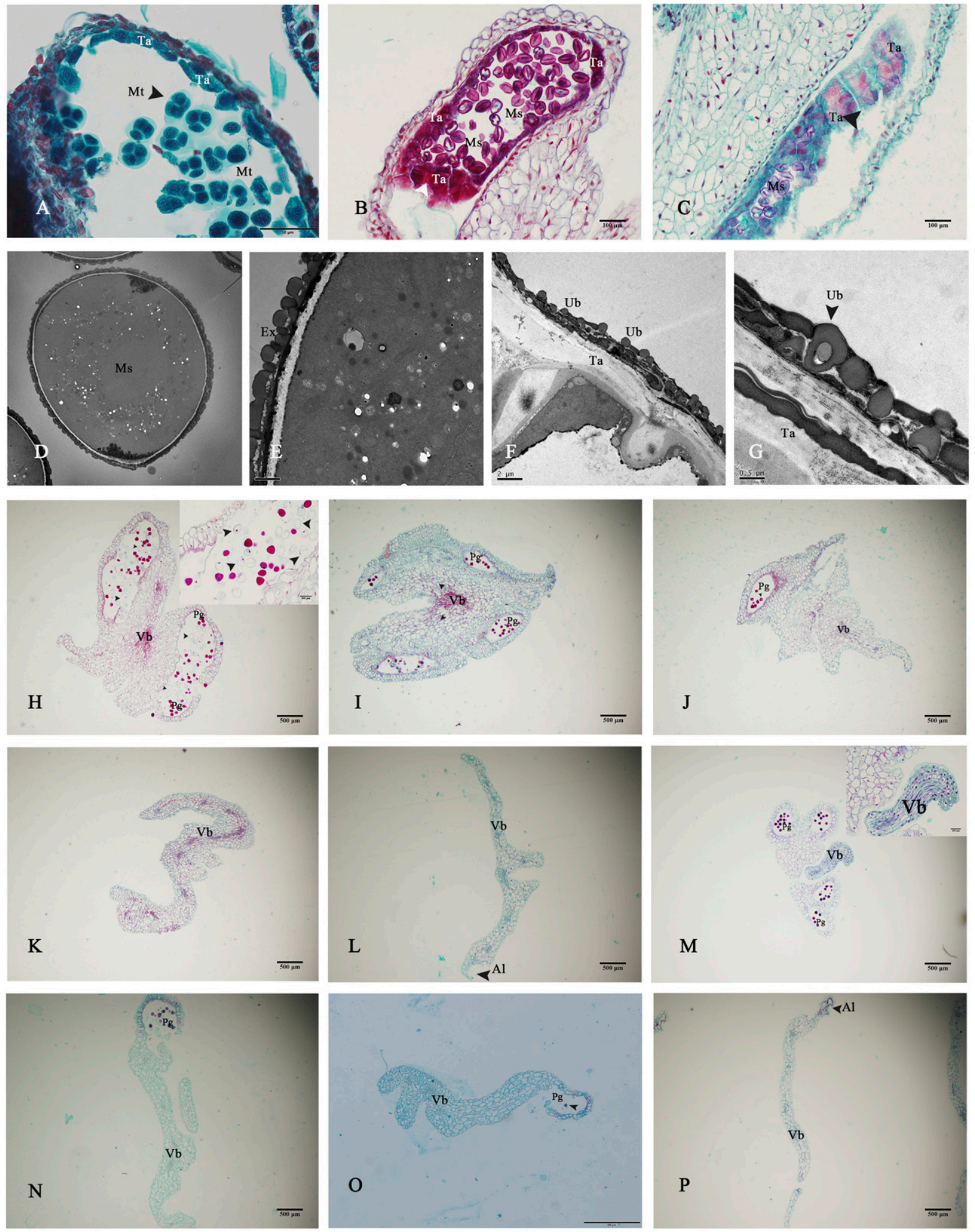

Fig. 4. Characterization of anther development in the Camellia oleifera male sterility line 'X1'. (A) Details of microspores showing tetrahedral microspore tetrads enclosed in the callose. (B) The cells of the tapetum were hypertrophic during the early microspore stage. (C) Details of the microspore period showing that the tapetum compressed the microspores. (D) Details of microspores under transmission electron microscope (TEM) observation. (E) Greater magnification of D showing the pollen exine. (F) TEM observation of an intact tapetum that did not disintegrate, with many Ubisch bodies attached to the surface of the cell membrane. (G) Greater magnification of $\mathbf{F}$ showing the Ubisch bodies. (H) Details of some empty pollen grains in an anther locule at the mature pollen stage. (I, J) Details of how the anther vascular bundle radial grew radially and how the area of the anther locule began to shrink. (K, L) Details of anther vascular bundle hyperplasia, during which the anther became a strip with empty anther locules. (M) Details of mature pollen grains in four anther chambers, and abnormal development of the vascular bundle with outward radial elongation. ( $\mathbf{N}, \mathbf{O})$ Abnormal development of the vascular bundle with the outward hyperplasia characteristic, with few mature pollen grains in one pollen sac. (P) Details of the shrunken anther locule, and the anther became a long strip with no mature pollen grains. Al, anther locule; Ex, exine; Mt, microspore tetrads; Ms, microspore; Pg, pollen grain; Ta, tapetum; Vb, vascular bundle; Ub, Ubisch body. 


\section{Discussion}

The pollen abortion period of male sterility in $C$. oleifera. Pollen abortion is the primary phenotype of CMS (Wang et al., 2018). The onset of pollen abortion is critical to understanding the mechanisms of control for male sterility in flowering plants (Xie et al., 2005). Currently, cytoplasmic sterility lines have been reported publicly for many crops, including oguCMS (Melendi et al., 2008), napCMS, tourCMS, polCMS, hauCMS, orf220CMS (Wang et al., 2018), and oxaCMS (Heng et al., 2018). Because the genes that control pollen abortion are different between sterility lines, pollen abortion periods differ as a result of variances in the timing of gene expression and gene function (Chen et al., 2013). Therefore, even in the same species, the pollen abortion period of male sterility from different sterility subgroups can differ. For example, male sterility plants of Brassica napus (Heng et al., 2018; Luo et al., 2018; Melendi et al., 2008), Capsicum annuum (Ji et al., 2014), S. melongena (Chen et al., 2013), and Brassica pekinensis (Guo et al., 2016) exhibit diverse abortion times. According to Laser and Lersten (1972), the abortion period for male sterility can be divided into three subtypes: the absence of a pollen sac, microsporocyte abortion, and uninucleate pollen abortion. In addition, Yang and Cao (1997) studied anther development in Brassica campestris ssp. chinensis Makino CMS and observed that the four anther chambers did not form during the archesporial cell differentiation period; therefore, this plant was classified as the "absence of pollen sac" type. Wang et al. (2001) also described a male sterility line of B. campestris ssp. pekinensis and found that anther development was blocked during the microsporocyte period and that meiosis was abnormal; thus, it was defined as a "microsporocyte abortion" type. Heng et al. (2018) found that the following occurred when the rape Ouxin A male sterility line was transferred to B. juncea: Pollen abortion in the sterility line occurred at the uninucleate stage, the microspores were vacuolated, and complete pollen abortion occurred at the pollen maturation stage. In our case, morphological abnormalities in C. oleifera ' $\mathrm{X} 1$ ' sterile anthers began in the developing microspores after microspore formation. The first feature noted was that the tapetum was abnormally thickened during the microspore stage. Moreover, the anther vascular bundles displayed abnormal structures, resulting in pollen abortion at the pollen maturation stage. Thus, in this male-sterile plant that was observed by microscopy, pollen abortion appeared to have occurred after the microspore stage. Similar results were reported for B. juncea by Heng et al. (2018).

The cytological characteristics of pollen abortion in male-sterile C. oleifera. Regarding the cytological mechanism of male sterility, many studies on higher plants have observed that pollen abortion is closely related to abnormal development of the tapetum (Jiang et al., 2015). The tapetum is the innermost sporophytic cell layer and it is in direct contact with the developing pollen in the anther locule. The tapetum transports necessary nutrients, metabolites, and enzymes, and provides pollen exine wall components to ensure normal microsporogenesis and pollen germination (Melendi et al., 2008; Pacini et al., 1985). Because the occurrence and degradation of the tapetum affect the development of microspores directly (Yui et al., 2003), the timeliness of tapetum breakdown is crucial for the development of mature pollen grains: Premature degeneracy or its retardation both lead to pollen abortion (Guo et al., 2016; Melendi et al., 2008). Many malesterile plants have been described as sharing some characteristics with petaloid-type CMS in C. oleifera. Xie et al. (2005) found that, after meiosis, the tapetal cells of the sterile anther in Chinese cabbage were swollen and significantly vacuolated, causing abnormal development of microspores and pollen abortion. In addition, Guo et al. (2016) stated that for the cytoplasmic sterility line 13A61 of Chinese cabbage, the tapetum exhibited abnormal swelling, eventually leading to anther abortion. In our study, we observed that the tapetum of normal fertile half-sib family 'Z720' plants belonged to the glandular type; they began to disintegrate at the early microspore stage. The presence of an orbicular membrane connected the Ubisch bodies after degeneration of the tapetum. Finally, the tapetum disappeared when the pollen grains were mature. However, in the petaloid-type male sterility ' $\mathrm{X} 1$ ' line, the tapetal cells were hypertrophic (Fig. 4B and C), with inward radial elongation at the microspore stage, and they then compressed the microspores. Expansion of the tapetum may have consumed a large amount of nutrients, and the tapetum did not begin to disintegrate at the early microspore stage. Although some of the pollen exine formed normally, the microspores could not obtain enough nutrients, which may have been the cause of most of the empty pollen grains (Fig. 4H). In general, it has been suggested that an abnormal tapetum cannot supply sufficient nutrients for pollen development. Our findings are similar to those described in other species (Luo et al., 2018; Xie et al., 2005; Xie et al., 2018; Yui et al., 2003), whereby male sterility was associated with interference of the tapetum developmental pathway.

In higher plants, abnormal development of the anther vascular bundle is also one of the factors leading to male sterility (Laser and Lersten, 1972). This connective tissue is a banded structure located between the pair of microsporangia in the anther; it connects either directly to or surrounds the microsporangia (Jiang et al., 2007). The vascular bundle constitutes a channel for the anther to absorb water and transport nutrients. Indeed, abnormality of its structural differentiation and development might not only influence the normal development of microspores but also affects the timing of tapetum disintegration as a result of the decreased supply of certain physiologically active substances. Therefore, the vascular bundle is a key structure for microspore development in numerous plant species (Chen et al., 2013). Wang et al. (2001) reported that dysplasia of the anther vascular bundle was the cause of pollen abortion in $B$. campestris spp. pekinensis 88-3. Li et al. (2010) observed degeneration of the anther vascular bundle and connective tissue in Chrysanthemum morifolium, which led to pollen abortion. In addition, Chen et al. (2013) showed that in $S$. melongena, sterile anthers had abnormal structures and vascular bundle hyperplasia. In our study, we observed that abnormal anther vascular bundle development, such as inward and outward vascular bundle hyperplasia, occurred at the mature pollen stage in the sterile anther (Fig. 4I$\mathrm{P})$. This tendency might result in the gradual compression of the anther locule and a shrunken appearance of sterile anther locules. Finally, few mature pollen grains were observed in most of the sterile anthers of $C$. oleifera. Herein, we report that the long strip appearance of the anther in malesterile lines is likely a further consequence of abnormal anther vascular bundle behavior. This characteristic of petaloid-type CMS in C. oleifera corresponded well with the findings of a study by Chen et al. (2013).

\section{Conclusion}

In our study, we documented petaloidtype CMS in C. oleifera and described the anatomic abnormalities that occur during anther development. Our results suggest that pollen abortion in C. oleifera 'X1' initiates at the microsporocyte stage and lasts until the mature pollen stage. It is postulated that malfunctioning of the tapetum and anther vascular bundle are the major causes of pollen abortion. To clarify the petaloid-type CMS mechanism in C. oleifera, a more profound investigation of the physiological and molecular biological mechanisms is necessary.

\section{Literature Cited}

Chen, X., S. Luo, S. Xuan, C. Zhang, and S. Shen. 2013. Cytological observation of pollen abortion in eggplant male-sterile lines. J. Plant Genet. Resources 14:754-758 (in Chinese with English abstract).

Chen, Y., D. Wang, Y. Liu, S. Peng, L. Su, and L. Chen. 2011. Research on selection of superior male-sterile clone of Camellia oleifera. J. Central South Univ. For. Technol. 31:1-6 (in Chinese with English abstract).

Chen, X., H. Zhang, H. Sun, H. Luo, L. Zhao, Z. Dong, S. Yan, C. Zhao, R. Liu, C. Xu, S. Li, H. Chen, and W. Jin. 2017. Irregular pollen exine 1 is a novel factor in anther cuticle and pollen exine formation. Plant Physiol. 173: 307-325.

Gao, C., R. Yang, and D. Yuan. 2018. Structural characteristics of the mature embryo sac of Camellia oleifera. Nord. J. Bot. 36:E01673. 
Gao, C., D. Yuan, B. Wang, Y. Ya, D. Liu, and Z. Han. 2015a. A cytological study of anther and pollen development in Camellia oleifera. Genet. Mol. Res. 14:8755-8765.

Gao, C., D. Yuan, Y. Yang, B. Wang, D. Liu, and F. Zou. 2015b. Pollen tube growth and double fertilization in Camellia oleifera. J. Amer. Soc. Hort. Sci. 140:12-18.

Guo, Y., H. Cui, H. Li, and Y. Yue. 2016. Cytological investigation and breeding of CMS line in Chinese cabbage. J. Yunnan Agricultural Univ. (Nature Science) 31:427432 (in Chinese with English abstract).

Han, Z., D. Yuan, C. Li, Z. Zhu, and X. Zhang. 2015. Photosynthetic characteristics of Camellia yuhsienensis, Hu C. oleifera 'Huashuo' and superior individuals in their $\mathrm{F}_{1}$ hybrids. Nonwood For. Res. 33:9-13 (in Chinese with English abstract).

He, Y., G. Ning, Y. Sun, Y. Hu, X. Zhao, and M. Bao. 2010. Cytological and mapping analysis of a novel male sterile type resulting from spontaneous floral organ homeotic conversion in marigold (Tagetes erecta L.). Mol. Breed. 26:19-29.

Heng, S., S. Liu, C. Xia, H. Tang, F. Xie, T. Fu, and Z. Wan. 2018. Morphological and genetic characterization of a new cytoplasmic male sterility system (oxa CMS) in stem mustard (Brassica juncea). Theor. Appl. Genet. 131:59-66.

Ji, J., W. Huang, D. Li, Y. Yin, W. Chai, and Z. Gong. 2014. A CMS-related gene, Yatp6-2, causes increased ATP hydrolysis activity of the mitochondrial $\mathrm{F}_{1} \mathrm{~F}_{0}$-ATP synthase and induces male sterility in pepper (Capsicum annuum L.). Plant Mol. Biol. Rpt. 32:888-899.

Jiang, X., H. Wu, X. Lun, and D. Lu. 2007. Morphological characteristics and biological significance of specialized connectives in Lonicera (Caprifoliaceae). Acta Phytotaxonomica Sinica 45:39-51 (in Chinese with English abstract).

Jiang, J., Y. Wang, J. He, Y. Cai, and Y. Lin. 2015. Cytological studies of anther development of the double recessive genetic male sterile line, ms5ms6, of upland cotton (Gossypium hirsutum). Intl. J. Agr. Biol. 17:345-350.

Kozik, E.U., R. Nowak, M. Nowakowska, and B. Dyki. 2012. Leaves of sterility and morphological flowers differentiation of petaloid malesterile plants of carrot. J. Agr. Sci. 4:187-194.

Kumar, K.R., K.P. Singh, D.V.S. Raju, S. Panwar, R. Bhatia, P.K. Jain, and V. Kumar. 2017. Standardization of rapid multiplication protocol in petaloid male sterile lines of African marigold (Tagetes erecta) through in vitro culture. Indian J. Agr. Sci. 87:31-38.
Laser, K.D. and N.R. Lersten. 1972. Anatomy and cytology of microsporogenesis in cytoplasmic male sterile angiosperms. Bot. Rev. 38:425454.

Li, F., S. Chen, F. Chen, N. Teng, W. Fang, F. Zhang, and Y. Deng. 2010. Anther wall development, microsporogenesis and microgametogenesis in male fertile and sterile chrysanthemum (Chrysanthemum morifolium Ramat., Asteraceae). Scientia Hort. 126:261267.

Luo, C., Y. Sun, Y. Zhang, Y. Guo, M. Klima, and S. Hu. 2018. Genetic investigation and cytological comparison of two genetic male sterile lines 9012A and MSL in Brassica napus L. Euphytica 214:124.

Melendi, P.G., M. Uyttewaal, C.N. Morcillo, J.R. Mora, S. Fajardo, F. Budar, and M.M. Lucas. 2008. A light and electron microscopy analysis of the events leading to male sterility in OguINRA CMS of rapeseed (Brassica napus). J. Expt. Bot. 59:827-838.

Pacini, E., G.G. Franchi, and M. Hesse. 1985. The tapetum: Its form, function, and possible phylogeny in Embryophyta. Plant Syst. Evol. 149:155-185.

Qin, S., J. Rong, W. Zhang, and J. Chen. 2018. Cultivation history of Camellia oleifera and genetic resources in the Yangtze River Basin. Biodiversity Sci. 26(4):384-395 (in Chinese with English abstract).

Sun, S., X. Zhang, M. Ma, and W. Ning. 2016 Morphological and cytological study on male sterile dandelion (Taraxacum junpeianum Kitam.). J. Plant Genet. Resources 17:491496 (in Chinese with English abstract).

Tan, G., F. Wang, J. Ma, X. Zhang, and A. Xiong. 2017. Identification and analysis of the characteristics of a wild petaloid male-sterile carrot 'Wuye-BY'. J. Plant Genet. Resources 18:1216-1220 (in Chinese with English abstract).

Tian, H., A. Kuang, M.E. Musgrave, and S.D. Russell. 1998. Calcium distribution in fertile and sterile anthers of a photoperiod-sensitive genic male-sterile rice. Planta 204:183-192.

Wang, J., X. Ren, Y. Huang, X. An, T. Gao, and Q. Meng. 2018. Morphological and cytological characteristics of cytoplasmic male sterility system 09-05A in spring stem mustard (Brassica juncea var. tumida Tsen et Lee). J. Zhejiang Univ. (Agr. Life Sci.) 44:588-593 (in Chinese with English abstract).

Wang, F., C. Wang, and Z. Song. 2001. The cytological observation of pollen and anther development in the cabbage 88-3 male-sterile line. Acta Bot. Boreal.-Occident. Sin. 21:570 574 (in Chinese with English abstract).
Wen, Y., S. Su, L. Ma, S. Yang, Y. Wang, and X. Wang. 2018. Effects of canopy microclimate on fruit yield and quality of Camellia oleifera. Scientia Hort. 235:132-141.

Wolyn, D.J. and A. Chahal. 1998. Nuclear and cytoplasmic interactions for petaloid malesterile accessions of wild carrot (Daucus carota L.). J. Amer. Soc. Hort. Sci. 123:849-853.

Wright, J., A. Reilley, J. Labriola, S. Kut, and T. Orton. 1996. Petaloid male-sterile plants from carrot cell cultures. HortScience 31:421-425.

Xie, C., Y. Yang, Y. Qiu, X. Zhu, and H. Tian. 2005. Cytochemical investigation of genic male-sterility in Chinese cabbage. Sex. Plant Reprod., doi: 10.1007/soo497-005-0001-5.

Xie, H., X. Peng, M. Qian, Y. Cai, X. Ding, Q. Chen, Q. Cai, Y. Zhu, L. Yan, and Y. Cai. 2018. The chimeric mitochondrial gene orf 182 causes non-pollen-type abortion in Dongxiang cytoplasmic male-sterile rice. Plant J. 95:715-726.

Xiong, H., F. Zou, D. Yuan, X. Tan, J. Yuan, T. Liao, and G. Niu. 2019. Comparison of selfand cross-pollination in pollen tube growth, early ovule development and fruit set of $\mathrm{Ca}$ mellia grijsii. Intl. J. Agr. Biol. 21:819-826.

Xiong, H., F. Zou, D. Yuan, X. Zhang, and X. Tan. 2016. Orthogonal test design for optimizing the culture medium for in vitro pollen germination of feijoa (Acca sellowiana cv. Unique). N. Z. J. Crop Hort. Sci. 44:192-202.

Yang, X. and S. Cao. 1997. Cytomorphological research on anther development of pol CMS in non-heading Chinese cabbage (Brassica canpestris L. ssp. chinensis Makino). J. Nanjing Agr. Univ. 20:36-43 (in Chinese with English abstract).

Yu, X., Y. Liu, Y. Lv, Z. Liu, Z. Chen, G. Lu, and J. Cao. 2014. Development of molecular markers specific to petaloid-type cytoplasmic male sterility in tuber mustard (Brassica juncea var. tumida Tsen et Lee). Mol. Biol. Rep. 41:769778.

Yu, X., H. Lu, G. Lu, Z. Chen, J. Cao, and Y. Hirata. 2010. Analysis of genetic diversity in cytoplasmic male sterility, and association of mitochondrial genes with petaloid-type cytoplasmic male sterility in tuber mustard (Brassica juncea var. tumida Tsen et Lee). Mol. Biol. Rep. 37:1059-1067.

Yui, R., S. Iketani, T. Mikami, and T. Kubo. 2003. Antisense inhibition of mitochondrial pyruvate dehydrogenase E1 $\alpha$ subunit in anther tapetum causes male sterility. Plant J. 34:57-66.

Zou, F., S. Chen, D. Yuan, R. Zhang, L. Zhang, and H. Xiong. 2016. Microsporogenesis, megasporogensis and male and female gametophyte development in Feijoa sellowiana (Myrtaceae). Intl. J. Agr. Bilol. 18:637-642. 